\title{
THE PLANAR FILAMENT EQUATION
}

\author{
Joel Langer and Ron Perline \\ Dept. of Mathematics, Case Western Reserve University \\ Dept. of Mathematics and Computer Science, Drexel University
}

\begin{abstract}
The planar filament equation and its relation to the modified Korteweg-deVries equation are studied in the context of Poisson geometry. The structure of the planar filament equation is shown to be similar to that of the 3-D localized induction equation, previously studied by the authors.
\end{abstract}

Remarks: The authors would appreciate it if anyone who downloads this file from the nonlinear science preprint archives would send an acknowledgement to: rperline@mcs.drexel.edu. We are interested in tracking the archive usage. Thanks!

Introduction. In this paper we study the planar filament equation

$$
\gamma_{t}=\frac{1}{2} \kappa^{2} T+\kappa_{s} N,
$$

where $\gamma(s, t)$ denotes an evolving planar curve, parameterized by arclength $s, \kappa$ is its curvature, $T$ its unit tangent, and $N$ its unit normal. $(\mathrm{PF})$ is a particular example of a geometric evolution equation on planar curves. By this we mean an evolution equation such that the velocity of any point on the curve is given in terms of the curve's geometric invariants. Such equations have been studied in a variety of applied contexts, as well 
as for their intrinsic geometric interest. The equation $\gamma_{t}=V_{0}(1-\epsilon \kappa) N$ appears in combustion theory and crystal growth $([\mathrm{Lan}],[\mathrm{M} 1 \mathrm{r}])$. The curve shortening flow $\gamma_{t}=\kappa N$ has recently been the focus of intensive study by geometers (see, for example [Ga] and $[\mathrm{Ga}-\mathrm{H}])$. One impetus for its study has been its relation to the construction of geodesics in Riemannian geometry; but other recent research has been concerned with the analytical properties of the flow on curves in $R^{2}$, where of course the study of geodesics is not an issue. The curve shortening flow can be thought of as (minus) the gradient flow of the length functional $\mathcal{L}(\gamma)=\int_{\gamma} d s$ on the appropriate space of curves. As we shall see, (PF) can be viewed as a Hamiltonian evolution equation, with $\mathcal{L}$ as the Hamiltonian. Thus $(\mathrm{PF})$ is a very natural equation to study. (PF) has already appeared in the mathematical literature in several contexts. It appears implicitly in $[\mathrm{Ch}-\mathrm{T}]$, which is a discussion of integrable foliations by curves of surfaces of constant curvature. Also, in [La 1-2], (PF) is mentioned as one of a list of evolution equations of curves related to nonlinear evolution equations solved by the two-component inverse-scattering method. For a more recent discussion, see $[\mathrm{G}-\mathrm{P}]$. All of these authors point out the close connection between (PF) and the modified Korteweg-deVries equation

$(\mathrm{mKdV})$

$$
u_{t}=u_{s s s}+\frac{3}{2} u^{2} u_{s}
$$

one of the well known examples of a completely integrable equation; we will later describe the connection in detail. In this paper, we wish to emphasize the integrability of (PF) itself and think of $(\mathrm{PF})$ as the geometric realization of $(\mathrm{mKdV})$; somewhat facetiously, 
$(\mathrm{PF})$ is "mKdV for geometers." (PF) may well have the distinction of being the simplest geometric evolution equation which is integrable.

Our interest in $(\mathrm{PF})$ came from an observation made in the course of our recent work on the localized induction equation

$$
\gamma_{t}=\kappa B
$$

Here, $\gamma(s, t)$ is (for fixed $t$ ) a space curve parameterized by arclength $s$, with curvature $\kappa$, torsion $\tau$ and Frenet frame $T, N, B$. (LIE) is a simplified model of vortex filament evolution in fluid mechanics. (LIE) was known to be Hamiltonian on the appropriate space of curves $([\mathrm{M}-\mathrm{W}])$. In [L-P 2], we established that (LIE) is in fact a completely integrable Hamiltonian equation; in particular, there exists an infinite sequence of commuting vectorfields, beginning with (LIE). We list the first few such vectorfields:

$$
\begin{aligned}
X_{-2} & =\kappa B \\
X_{-1} & =\frac{1}{2} \kappa^{2} T+\kappa^{\prime} N+\kappa \tau B, \\
X_{0} & =\kappa^{2} \tau T+\left(2 \kappa^{\prime} \tau+\kappa \tau^{\prime}\right) N \\
& +\left(\kappa \tau^{2}-\kappa^{\prime \prime}-\frac{1}{2} \kappa^{3}\right) B, \\
X_{1} & =\left(-\kappa \kappa^{\prime \prime}+\frac{1}{2}\left(\kappa^{\prime}\right)^{2}+\frac{3}{2} \kappa^{2} \tau^{2}-\frac{3}{8} \kappa^{4}\right) T \\
& +\left(-\kappa^{\prime \prime \prime}+3 \kappa \tau \tau^{\prime}+3 \kappa^{\prime} \tau^{2}-\frac{3}{2} \kappa^{2} \kappa^{\prime}\right) N \\
& +\left(\kappa \tau^{3}-3\left(\kappa^{\prime} \tau\right)^{\prime}-\frac{3}{2} \kappa^{3} \tau-\kappa \tau^{\prime \prime}\right) B .
\end{aligned}
$$

Here ' denotes differentiation with respect to $s$. We note that (LIE) is the first vectorfield appearing in the sequence. The numbering system is explained in [L-P 2] and 
need not concern us here. What is of interest is that the odd vectorfields listed preserve planarity of curves. If $\gamma$ is planar, then of course $\tau(s)=0$ for all values of $s$. But in that case, the coefficients of $B$ in $X_{-1}$ and $X_{1}$ vanish by inspection. The resulting vectorfields only have tangential and normal components, thus preserving planarity of the initial curve. Also note that $X_{-1}$ restricted to planar curves is just (PF).

This line of reasoning suggests that perhaps all the odd vectorfields in our infinite list are planarity-preserving, and that this infinite sequence of vectorfields defined on planar curves, starting with (PF) itself, is an integrable system. In this paper, we illustrate the basic aspects of the structure of this integrable hierarchy, as well as its relation to the $(\mathrm{mKdV})$ hierarchy. It is also interesting to observe that (PF) "inherits" its structure from (LIE); upon occasion, we will refer to [L-P 2] for the aspects of the structure of the (LIE) hierarchy which we will find useful.

Structure of the planar filament equation. It is helpful to make precise the space of curves we shall be working with: We set $A L=\left\{\gamma:(-\infty, \infty) \rightarrow \mathbb{R}^{2}: \gamma\right.$ is arc-lengthparameterized, and is asymptotic to the $x$-axis $\}$. To say $\gamma$ is asymptotic to the $x$-axis means that there exist constants $\lambda_{+}, \lambda_{-}$such that

$$
\lim _{s \rightarrow \pm \infty}\left[s e_{1}-\gamma(s)\right]=\lambda_{ \pm} e_{1}
$$

where $e_{1}=(1,0)$. 
Within $A L$, there is a distinguished subspace of balanced asymptotically linear curves $B A L=\left\{\gamma \in A L: 0=\Lambda_{+}=\lambda_{+}+\lambda_{-}\right\}$. Elements of $B A L$ correspond to convenient parameterizations of asymptotically linear curves. This is reflected in the fact that there is a simple characterization of the tangent space to $B A L$ : if $\gamma \in B A L$ and $W=f T+g N$ is a vector field along $\gamma(T, N$ is the Frenet frame along $\gamma)$ then

$$
T_{\gamma} B A L=\{W: f=\mathcal{S}(g \kappa)\}
$$

where $\mathcal{S}$ denotes the antidifferentiation operator

$$
\mathcal{S}(f)(s)=\left(\frac{1}{2}\right)\left[\int_{-\infty}^{s} f(u) d u-\int_{s}^{\infty} f(u) d u\right]
$$

We now consider the curvature correspondence which assigns to a curve $\gamma \in B A L$ its curvature function $\kappa(s)$. Of course, this correspondence has been considered in many contexts; but it is worth noting that we can consider this a special case of the Hasimoto transformation (introduced in [Has2] and subsequently studied in [L-P 1-2]) which assigns to a space curve a complex function via the formula $\gamma \rightarrow \mathcal{H}(\gamma)=\kappa(s) e^{i \int \tau(u) d u}$ (for planar curves $\tau$ vanishes and we are just left with $\kappa$ ). It is for this reason that we denote the curvature correspondence for planar curves by $\mathcal{H}_{P}$, and informally refer to it as the planar Hasimoto transformation.

Our first task is to compute the differential of $\mathcal{H}_{P}$, considered as a map from $B A L$ to the space of functions. To do so, we recall some elementary formulas for the variation of geometric invariants along a curve ([L-P 2]): 
Proposition. Denote by $\gamma=\gamma(w, u):(-\epsilon, \epsilon) \times(a, b) \rightarrow \mathbb{R}^{3}$ a one-parameter family of planar curves. If $W=\frac{\partial \gamma}{\partial w}(0, u)$ is the variation vector field along $\gamma$, and $\gamma$ has speed $v=\left|\frac{\partial \gamma}{\partial u}\right|$, curvature $\kappa$, then $v$ and $\kappa$ vary according to:

$$
\begin{aligned}
& W(v)=<W^{\prime}, T>v=-\alpha v, \quad \alpha=-<W^{\prime}, T> \\
& W(\kappa)=\left\langle W^{\prime \prime}, N>-2<\kappa W^{\prime}, T>\right.
\end{aligned}
$$

Again, ' denotes derivative with respect to the arclength parameter along $\gamma$.

From formula (a), it is immediate that if $W=f T+g N$ has coefficients satisfying $f^{\prime}(s)=g(s) \kappa(s)$, then the variation of $v$ along $W$ is 0 ; we call such vector fields locally arclength preserving, for obvious reasons. Observe that elements of $T_{\gamma} B A L$ satisfy this condition.

Now let $\gamma$ be an element of $B A L$, and $W \in T_{\gamma} B A L$. Formula (b) gives a simple formula for the variation of $\kappa$ along $W$ :

$$
W(\kappa)=d \mathcal{H}_{P}(W)=\left\langle W^{\prime \prime}, N>\right.
$$

thus giving a formula for the differential of the planar Hasimoto transformation.

As a first application, let $W=\frac{1}{2} \kappa^{2} T+k^{\prime} N$ which is obviously in $T_{\gamma} B A L$. Then by the Frenet equations, we obtain :

$$
\begin{aligned}
W^{\prime} & =\left(\kappa^{\prime \prime}+\frac{1}{2} \kappa^{3}\right) N, \\
\text { thus } d \mathcal{H}_{P}(W) & =<W^{\prime \prime}, N>=\kappa^{\prime \prime \prime}+\frac{3}{2} \kappa^{2} \kappa^{\prime}
\end{aligned}
$$

An immediate consequence of this last calculation is the relation of $(\mathrm{PF})$ to $(\mathrm{mKdV})$ : if we have a solution $\gamma(s, t)$ to (LIE), then $\kappa(s, t)$ satisfies (mKdV). Conversely, given a 
solution $\kappa(s, t)$ to $(\mathrm{mKdV})$, we can reconstruct the unit tangent vector $T(s, t)$ to $\gamma$ by computing $\theta=\int^{s} \kappa d s$, and setting $T=(\cos (\theta), \sin (\theta))$; the curve $\gamma$ itself is obtained by one further antidifferentiation of $T$.

Soliton solutions for $(\mathrm{mKdV})$ are well-known ([Hir] $)$; these give us explicit formulas for $\kappa(s, t)$. At the end of this paper, we show plots of an evolving 2-soliton curve with curvature $\kappa$ satisfying $(\mathrm{mKdV})$, as viewed from a moving frame of reference so that the "lowest" point of the curve is centered with respect to the viewing box.

Going back to the formula for the differential of the planar Hasimoto map, we interpret this formula in geometric terms. Let us return for a moment to the localized induction equation. The (LIE) hierarchy enjoys a number of properties common to integrable systems. In particular, there is a linear recursion operator $\mathcal{R}$ which generates the commuting vector fields and is given by the formula

$$
\mathcal{R} W=-\mathcal{P}\left(T \times W^{\prime}\right) \text {, where } W=f T+g N+h B \text { and } \mathcal{P} W=\mathcal{S}(g \kappa) T+g N+h B
$$

By stating that $\mathcal{R}$ generates the (LIE) hierarchy, we mean more precisely that $X_{k}=$ $\mathcal{R}^{k+2} X_{-2}, k=0,1,2, \ldots$; this is proved in [L-P 2] and the reader can easily check the first few instances of this formula by considering the list of $X_{i}$ already presented from the (LIE) hierarchy.

One can compute $\mathcal{R}^{2} W$ to obtain

$$
\mathcal{R}^{2} W=-\mathcal{P}\left(W^{\prime \prime}+\mathcal{S}\left(<W^{\prime}, \kappa B>\right) \kappa B\right) .
$$


If we now consider a planar curve $\gamma$ and a planar field $W$ along $\gamma$, this formula simplifies to $\mathcal{R}^{2} W=-\mathcal{P}\left(W^{\prime \prime}\right)$. To complete the connection with the differential for the planar Hasimoto map, we introduce the operator $\mathcal{Z}$ mapping

$$
\mathcal{Z}: W \rightarrow<W, N>
$$

Then we observe that $d \mathcal{H}_{P}(W)=-\mathcal{Z R}^{2}(W)$.

As just stated, we know that $\mathcal{R}$ satisfies $\mathcal{R}^{2} X_{k}=X_{k+2}, k=-2,-1, \ldots$. From the formula for $\mathcal{R}^{2}$ given above, it is clear that if $\gamma$ is a planar curve with a planar field $W=f T+g N$ along it, then $\mathcal{R}^{2}$ preserves the planarity of $W$. In particular, the field $X_{-1}=\frac{\kappa^{2}}{2} T+\kappa_{s} N+\kappa \tau B$ restricts to $\frac{\kappa^{2}}{2} T+\kappa_{s} N$ along a planar curve $\gamma$, and hence all the odd flows in (LIE) hierarchy preserve planarity (The fact that these planar vectorfields commute follows immediately from the fact that they are restrictions of commuting vectorfields defined along space curves). We call the restriction of $\mathcal{R}^{2}$ to planar vectorfields along planar curves the planar recursion operator and denote it by $\mathcal{R}_{P}$. We can now express the differential formula for the planar Hasimoto transformation via $d \mathcal{H}_{P}(W)=-\mathcal{Z} \mathcal{R}_{P}(W)$.

Now that we have demonstrated that the sequence of vectorfields $X_{-1}, X_{1}, X_{3}, \ldots$ induce evolution equations on planar curves, we can discuss their hamiltonian nature. As is well known (see $[\mathrm{F}-\mathrm{T}]$ ) there exist an infinite sequence of conserved quantities for the $(\mathrm{mKdV})$ hierarchy, defined in terms of integrals of polynomials in $u$ and its derivatives:

$$
\tilde{I}_{0}=\int \frac{1}{2} u^{2} d s, \quad \tilde{I}_{2}=\int\left(\frac{1}{2}\left(u^{\prime}\right)^{2}-\frac{1}{8} u^{4}\right) d s, \ldots
$$


Given the relation between $(\mathrm{PF})$ and $(\mathrm{mKdV})$ previously demonstrated, it is a simple consequence of the chain rule that these conserved quantities "pull back" to give invariants for $(\mathrm{PF})$ (simply replace $u$ by $\kappa$ ).

These invariants for $(\mathrm{PF}): I_{0}=\int \frac{1}{2} \kappa^{2} d s, \quad I_{2}=\int\left(\frac{1}{2}\left(\kappa^{\prime}\right)^{2}-\frac{1}{8} \kappa^{4}\right) d s, \ldots$ are closely related the invariants $I_{i}$ of the (LIE) hierarchy discussed in [L-P 2]: they are the restriction to planar curves of the even invariants of (LIE); the odd invariants, restricted to planar curves, vanish. Again invoking [L-P 2], we know that the full (LIE) hierarchy can be put in hamiltonian form using these invariants: $X_{i}=J \nabla I_{i}$, where the $I_{i}$ are the invariants of the (LIE) hierarchy, $\nabla$ denotes formal $L^{2}$ gradient, and $J$ denotes the skew operator $J(W)=-\mathcal{P}(T \times W)$. But the (LIE) vectorfields are also hamiltonian with respect to a second operator: $X_{i}=K \nabla I_{i-1}$, where $K=J(d / d s) J$. If $\gamma$ is planar with planar field $W=f T+g N$, then $K(W)=\mathcal{S}\left(\kappa g^{\prime}\right) T+g^{\prime} N$. In particular, $X_{2 k+1}=K \nabla I_{2 k}, k=0,1,2, \ldots$ From our previous discussion, all the objects in this last equation make sense for planar curves: $I_{2 k}$ restricts nontrivially to planar curves, with gradient involving components in the $T$ and $N$ directions; $K$ preserves planarity; and the $X_{2 k+1}$ are also defined on planar curves.

This demonstrates the hamiltonian nature of all the vectorfields in the (PF) hierarchy, except $(\mathrm{PF})$ itself! This is because the hamiltonian for $(\mathrm{PF})$ is not one of the invariants induced by $(\mathrm{mKdV})$, but rather the (renormalized) length functional $\mathcal{L}$ which can be defined on our space of curves $B A L$ via the formula $\mathcal{L}(\gamma)=\lambda_{+}-\lambda_{-}$(If we had been dealing with periodic curves, then the hamiltonian would have been the ordinary length 
functional). One can show that $\nabla \mathcal{L}=-\kappa N$ (as one would expect from the ordinary length functional) and $K \nabla \mathcal{L}=-\left(\frac{1}{2} \kappa^{2} T+\kappa^{\prime} N\right)$. Thus, up to a minus sign, we obtain $(\mathrm{PF})$.

Finally, we discuss how one can interpret $\mathcal{H}_{P}$ as a Poisson map. To do so, we recall ([F-T], [L-P 2]) that $(\mathrm{mKdV})$ is also hamiltonian; it can be written as $u_{t}=\tilde{K} \nabla F$, where $\tilde{K}$ is the skew operator $\frac{d}{d s}$, and $F$ is just (minus) $\tilde{I}_{2}$. The (mKdV) hierarchy of commuting evolution equations is generated by the recursion operator $\tilde{\mathcal{R}}(u, v)=v^{\prime \prime}+u^{2} v^{\prime}+u^{\prime} \mathcal{S}(u v)$ (in this discussion, our notational conventions are different from those we used in [L-P 2]). There exists an infinite sequence of Poisson brackets associated with $\tilde{K}$ and $\tilde{\mathcal{R}}$ : For any two functionals $F=\int f\left(u, u^{\prime}, u^{\prime \prime}, \ldots\right) d s$ and $G=\int g\left(u, u^{\prime}, u^{\prime \prime}, \ldots\right) d s$, define $\{F, G\}_{k}=<\tilde{\mathcal{R}}^{k} \tilde{K} \nabla F, \nabla G>, k=0,1,2, \ldots$ These Poisson brackets are compatible with the $(\mathrm{mKdV})$ hierarchy in that all of the invariants $I_{2 k}$ of the $(\mathrm{mKdV})$ hierarchy commute with respect to all of the Poisson brackets just listed. Similarly, for functionals on planar curves one can define the Poisson bracket $\{F, G\}_{P}=\langle K \nabla F, \nabla G>$. As a simple corollary of our formula for the differential of $\mathcal{H}_{P}$ in terms of $\mathcal{R}_{P}$, one can show that $\mathcal{H}_{P}$ is Poisson with respect to the two brackets $\{,\}_{2}$ and $\{,\}_{P}$.

In this paper, we have reconsidered the equivalence of the planar filament equation and $(\mathrm{mKdV})$; this equivalence is realized via the elementary geometric transformation of assigning to a planar curve its curvature function. In the theory of integrable systems, there are a number of examples of interesting pairs of integrable systems which can be 
shown to be equivalent: the Kepler problem and geodesic flow on the sphere; the Neumann problem and geodesic flow on an ellipsoid; the localized induction equation and the cubic non-linear Schrödinger equation (see [Moser],[Has]). In each case the transformation relating the two systems is a natural one from the point of view of differential geometry. It would be of great interest to have a unifying theory which could "explain" the existence of these several examples. 
Soliton curve at $t=-.7$

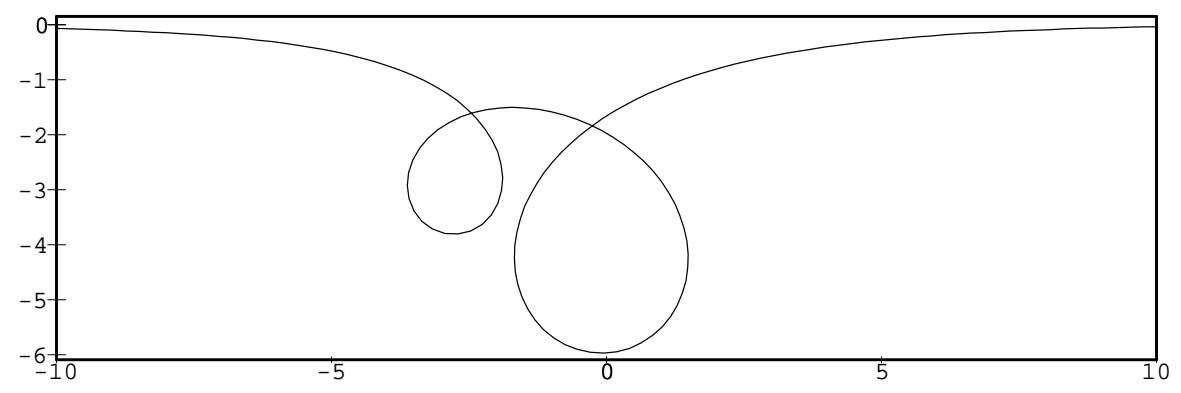

Soliton curve at $t=-.3$

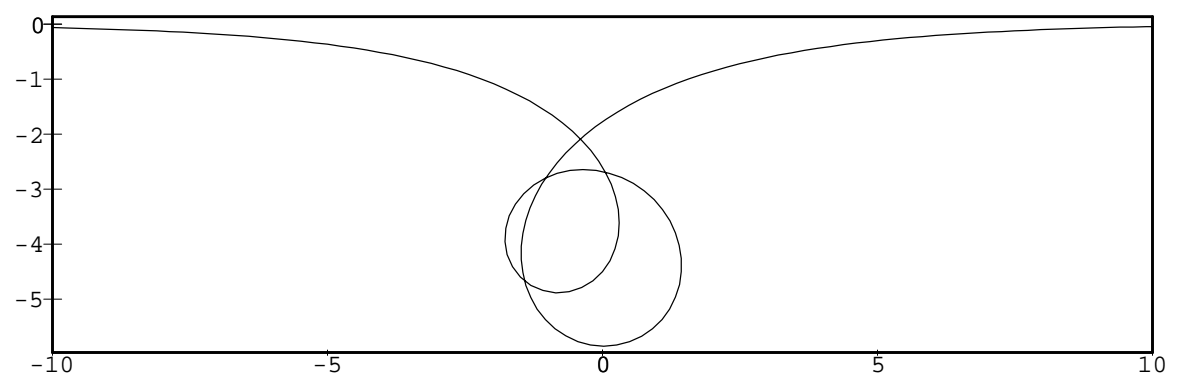




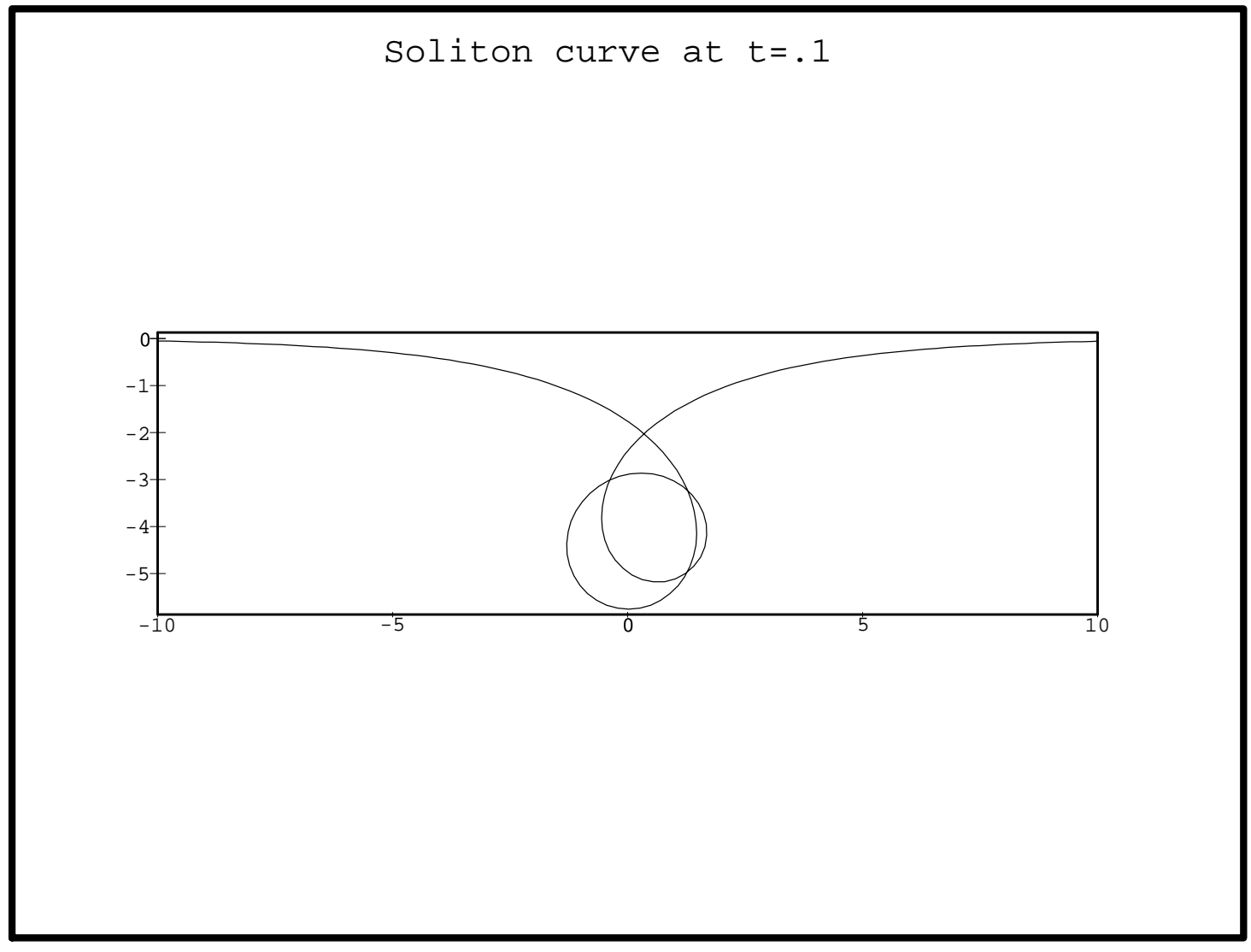

Soliton curve at $t=.5$

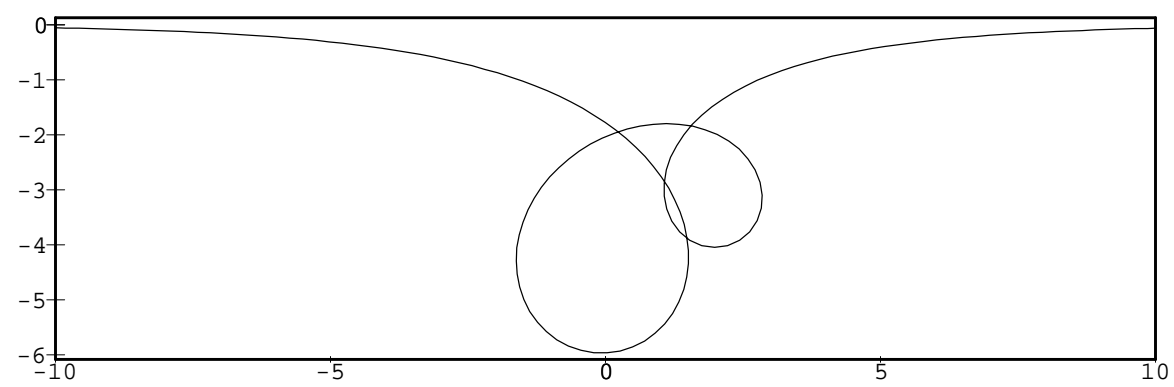




\section{REFERENCES}

[Ba] G.K. Batchelor, An introduction to fluid dynamics, Cambridge University Press, New York, 1967.

[Ch-T] S.S. Chern and K. Tenenblat, Foliations on a surface of constant curvature and modified KdV equations, J. Diff. Geom 16 (1981), 347.

[F-T] L. Faddeev and L. Takhtajan, Hamiltonian methods in the theory of solitons, Springer-Verlag, Berlin, 1980.

[Ga] M. Gage, curve shortening makes convex curves circular, Invent. Math. 76 (1986), 357.

[Ga-H] M. Gage and R. Hamilton, The heat equation shrinking convex plane curves, J. Diff. Geom. 23 (1986), 69.

[G-P] R. Goldstein and D. Petrich, The Korteweg-deVries hierarchy as dynamics of closed curves in the plane, Physical Review Letters 67 (23) (1991), 3203.

[Has 1] H. Hasimoto, Motion of a vortex filament and its relation to elastica, J. Phys. Soc. Japan 31 (1971), 293

[Has 2] — A soliton on a vortex filament, J. Fluid Mech. 51 (1972), 477.

[Hir] R. Hirota, Exact solution of the modified Korteweg-de Vries equation for multiple collisions of solitons, J. Phys. Soc. Japan 33(5) (1972), 1456.

[La 1] G.L. Lamb, Elements of soliton theory, Wiley Interscience, New York, 1980.

[La 2] _ Solitons on moving space curves, J. Math. Phys. 18 (1977), 1654.

[Lan] J.S. Langer, Instabilites and pattern formation in crystal growth, Rev. Modern Phys. 52 (1980), 1

[L-P 1] J. Langer and R. Perline, The Hasimoto transformation and integrable flows on curves, Appl. Math. Lett. 3(2) (1990), 61.

[L-P 2] _ Poisson geometry of the filament equation, J. Nonlinear Sci. 1 (1991), 71.

[Mar] G. Markstein, Experimental and theoretical studies of frame front stability, J. Aero. Sci. 18 (1951), 199.

[M-W] J. Marsden and A. Weinstein, Coadjoint orbits, vortices, and Clebsch variables for incompressible fluids, Physica 7D (1983), 305.

[Mos] J. Moser, Dynamical Systems C.I.M.E. Lectures, Birkhäuser, Boston, 1980. 